https://helda.helsinki.fi

\title{
The Association Between Social Support, Body Mass Index and Increased Risk of Prediabetes : the Cardiovascular Risk in Young Finns Study
}

Serlachius, Anna

2017-04

Serlachius , A, Elovainio, M , Juonala , M , Shea, S , Sabin , M , Lehtimäki , T , Raitakari , O , Keltikangas-Jarvinen , L \& Pulkki-Raback , L 2017 , ' The Association Between Social Support, Body Mass Index and Increased Risk of Prediabetes : the Cardiovascular Risk in Young Finns Study ', International Journal of Behavioral Medicine, vol. 24 , no. 2 , pp. 161-170 . https://doi.org/10.1007/s12529-016-9597-0

http://hdl.handle.net/10138/236698

https://doi.org/10.1007/s12529-016-9597-0

publishedVersion

Downloaded from Helda, University of Helsinki institutional repository.

This is an electronic reprint of the original article.

This reprint may differ from the original in pagination and typographic detail.

Please cite the original version. 


\title{
The Association Between Social Support, Body Mass Index and Increased Risk of Prediabetes: the Cardiovascular Risk in Young Finns Study
}

\author{
Anna Serlachius ${ }^{1,2}$ (D) Marko Elovainio ${ }^{1,3} \cdot$ Markus Juonala $^{4,5} \cdot$ Steven Shea $^{2,6}$. \\ Matthew Sabin $^{7,8}$. Terho Lehtimäki ${ }^{9,10}$ - Olli Raitakari ${ }^{4,11}$. \\ Liisa Keltikangas-Järvinen ${ }^{1}$ • Laura Pulkki-Råback ${ }^{1,12}$
}

Published online: 3 October 2016

(C) International Society of Behavioral Medicine 2016

\begin{abstract}
Purpose The psychosocial determinants of prediabetes are poorly understood. The aims of our study were (1) to analyse the association between perceived social support in young adulthood and fasting glucose levels and prediabetes in midadulthood in a cohort of healthy Finns, (2) to explore whether body mass index (BMI), inflammation or depression mediate
\end{abstract}

Anna Serlachius

anna.serlachius@helsinki.fi

1 Institute of Behavioural Sciences, The University of Helsinki, P.O. Box 9, 00014, Helsinki, Finland

2 The Department of Medicine, Division of General Medicine, Columbia University, New York City, NY, USA

3 Institute for Health and Welfare, Helsinki, Finland

4 Research Centre of Applied and Preventive Cardiovascular Medicine, University of Turku, Turku, Finland

5 The Division of Medicine, Turku University Hospital, Turku, Finland

6 The Department of Epidemiology, Joseph Mailman School of Public Health, Columbia University, New York City, NY, USA

7 The Department of Paediatrics, University of Melbourne, Melbourne, Australia

8 Murdoch Childrens Research Institute, Royal Children's Hospital, Melbourne, Australia

9 The Department of Clinical Chemistry, Fimlab Laboratories, Tampere, Finland

10 School of Medicine, University of Tampere, Tampere, Finland

11 The Department of Clinical Physiology and Nuclear Medicine, Turku University Hospital, Turku, Finland

12 The Collegium for Advanced Studies, University of Helsinki, Helsinki, Finland this relationship, (3) and to examine the association between social support trajectory groups and fasting glucose.

Method A prospective design was used with an analytic sample of 1250 participants aged 3-18 years at baseline (1980) and aged 12-39 years when social support was measured. Fasting glucose and prediabetes were assessed 32 years after baseline. Linear and logistic regression was used to examine the association between social support and the outcome measures. A bootstrapping technique was used to examine mediation effects.

Results Social support was associated with future glucose levels in women after adjusting for childhood socioeconomic status (SES) and youth depression $(\beta=-0.136, p=0.001)$ and also predicted prediabetes in women after adjusting for childhood SES ( $\beta=1.31,95 \%$ CI 1.02 to $1.69, p=0.031)$. Both associations were attenuated after adjusting for BMI in midadulthood. BMI was found to mediate the relationship between social support and prediabetes in women $(\beta$ for indirect effect $\beta=0.09, \mathrm{SE}=0.03, \mathrm{CI}=0.03$ to 0.16 ).

Conclusion Low perceived social support in young adulthood is associated with high fasting glucose and prediabetes in midadulthood in women but not men. The association between social support and prediabetes in women can be partly explained by BMI.

Keywords Fasting glucose $\cdot$ Prediabetes $\cdot$ Prospective study Social support · Type 2 diabetes

\section{Introduction}

Type 2 diabetes mellitus is increasing worldwide at an alarming rate with total diabetes cases estimated to be as high as 366 million by 2030 [1]. As well as the growing epidemic 
of type 2 diabetes, the rates of people with prediabetes are increasing [2]. Impaired fasting glucose and impaired glucose tolerance are prediabetic states which predispose to later type 2 diabetes. They represent states of hyperglycemia but not to levels which are needed for a diagnosis of T2DM. Research has found that people with prediabetes are likely to go on to develop T2DM and are also at risk for cardiovascular complications [3].

Well-known risk factors for type 2 diabetes include family history of diabetes, older age, physical inactivity and overweight/obesity. In contrast, the psychosocial antecedents of prediabetes or type 2 diabetes, and in particular the underlying mechanisms by which they influence health outcomes, are not well understood. Evidence has accrued that psychosocial factors such as low socioeconomic status [4], depression [5] and work-related stress [6] are associated with an increased risk for type 2 diabetes, but prospective studies assessing the predictive role of social support are limited. This is despite the wellestablished association between social support and cardiovascular disease [7], which shares a similar aetiology to diabetes.

A small number of prospective studies have shown that social support is implicated in type 2 diabetes. Social support is usually separated into its structural components (the social network) and its functional components (the types of social support received and the perception/satisfaction with this support). Most of these prospective studies have examined structural social support, and the findings have been mixed, in particular regarding sex [8, 9]. For example, in a populationbased sample from Germany, men who lived alone were at higher risk for type 2 diabetes compared to men who cohabited; however, this association was not found for women [9]. In contrast, a study of Swedish adults found that higher levels of social integration (measured by examining the quantitative characteristics of the extended social network) reduced the risk for developing type 2 diabetes in women but actually increased this risk in men [8]. As well as the mixed findings regarding sex, there is a lack of studies exploring the pathways underlying the association between social support and type 2 diabetes. This gap in the research extends to the social support-health literature in general [10].

It has been suggested that low social support could directly affect type 2 diabetes through its impact on the physiological stress response and neuroendocrine system, resulting in excess secretion of glucocorticoids that increase insulin resistance and visceral obesity [11]. Studies have also shown that structural aspects of social relationships (such as social isolation) have been associated with increased stress-related inflammation and metabolic disturbances [12]. Research has also examined the association between social support and obesity, a significant risk factor for type 2 diabetes, with findings demonstrating that different types of social networks can both benefit and hurt weight loss efforts [13] and that lower levels of perceived support are associated with central adiposity [14,
15]. Other factors which may be implicated in the association between social support and type 2 diabetes include depression, as low social support has also been associated with a higher risk for depression [16], another risk factor for type 2 diabetes [5]. In contrast, high social support has been associated with adaptive coping strategies [17], which may help to prevent or lower the exposure to stress [18], as well as health behaviours which may protect against diabetes [19]. For a more comprehensive review on the association between stress, coping and social support, please refer to Thoits [17].

Due to these limitations in the literature, in particular the lack of studies exploring prospective mechanisms of the social support-diabetes link and the lack of studies assessing the role of functional social support, we had three main objectives: (1) to examine the association between perceived social support in young adulthood and fasting glucose levels and prediabetes in mid-adulthood in a cohort of healthy Finns, (2) to examine possible intermediate mechanisms, including the role of body mass index (BMI), inflammation and depression and (3) to examine social support trajectory groups and their association with fasting glucose.

\section{Methods}

\section{The Young Finns Study}

The Cardiovascular Risk in Young Finns Study (Young Finns Study) is an ongoing prospective study of cardiovascular health in a randomly selected cohort of Finns which began in 1980. The aim of the Young Finns Study was to assess the levels of coronary heart disease risk factors and their determinants in children and adolescents. The baseline assessment took place in 1980 with 3596 participants aged 3 to 18 years who were randomly selected from the national register of five regional areas in Finland. Follow-up assessments have been held every 3-5 years, with the latest in 2012.

\section{Study Population}

The current study participants are from the Young Finns Study cohort. The study participants were aged between 3 and 18 years at baseline (in 1980). The analytic sample consists of participants with data on perceived social support collected between 1989 and 2001 (when participants were aged 1239 years old) and who have data on the outcome measures collected in the 2012 follow-up (aged between 35 and 49 years). From this sample, we excluded participants with type 1 diabetes $(n=19)$ as well as participants with type 2 diabetes or prediabetes before 2001 (when the last social support measure was assessed) $(n=32)$. This resulted in an analytic sample of 1250 participants (Fig. 1). 
Fig. 1 Recruitment, follow-up and outcome data for study participants. T1DM type 1 diabetes mellitus, T2DM type 2 diabetes mellitus

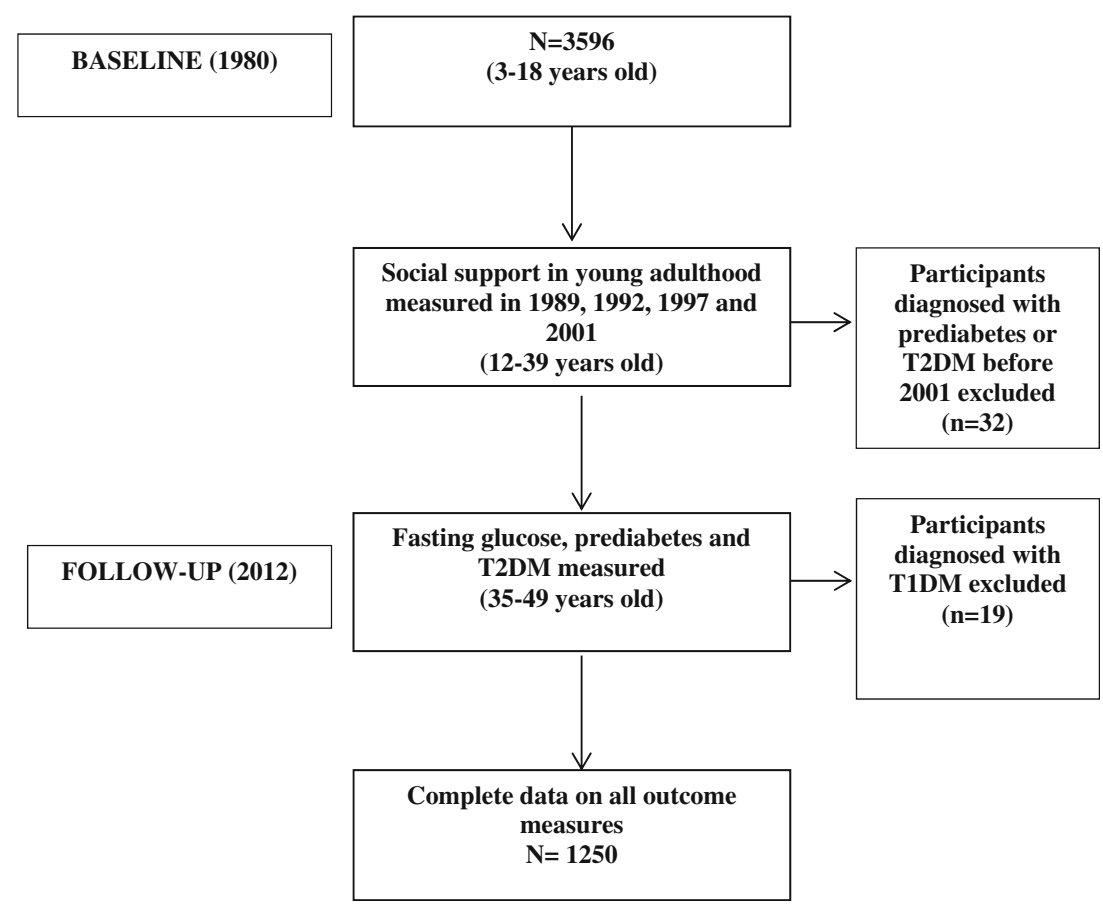

Written informed consent was obtained from all the participants. Ethics approval was granted from each participating university, and the study was conducted in accordance with the Helsinki Declaration.

\section{Outcome Measures}

The study measures were collected at clinical examinations and via survey methods as part of the long-term follow-up of the Young Finns cohort. Detailed methodology has been previously published [20].

\section{Fasting Glucose}

Serum fasting glucose concentrations were measured in 2012. Glucose concentrations were determined by the enzymatic hexokinase method (glucose reagent; Olympus, County Clare, Ireland).

\section{Prediabetes and Type 2 Diabetes}

Prediabetes was defined as fasting glucose 5.6-7.0 mmol/1 [21]. Type 2 diabetes was defined based on fulfilling one of the following criteria: (1) having a fasting plasma glucose of $\geq 7 \mathrm{mmol} / \mathrm{l}(\geq 125 \mathrm{mg} / \mathrm{dl})$, (2) having an HbAlc $\geq 6.5 \%$ ( $\geq 48 \mathrm{mmol} / \mathrm{l}$ ), (3) reporting a doctor's diagnosis or (4) taking medication for type 2 diabetes (as recorded from the Social Insurance Institution of Finland national registry).

Due to the low number of type 2 diabetes cases $(n=26)$, we combined type 2 diabetes with prediabetes cases for the analyses (total $n=285$ ) and refer to this combined group as the 'prediabetes' group in the results.

\section{Social Support in Young Adulthood}

Social support was measured by using the Perceived Social Support Scale-Revised [22] (functional social support). The mean score from four measurements taken in youth to young adulthood was combined (1989, 1992, 1997 and 2001) to retain a maximum number of participants. The correlations between the four time points ranged from 0.306 to 579 $(p<0.001)$. The scale consists of 12 items which are rated on a five-point Likert scale ranging from 1 to 5 ( $1=$ totally disagree, $5=$ totally agree). The items measure perceived social support from friends, family and significant others (e.g. 'I can talk about my problems with my friends' and 'There is a special person who is around when I am in need'). The scale was negatively skewed and demonstrated lack of linearity which we corrected by reversing the scale (to correct for a negative skew) and then applied a square root transformation (to correct for lack of linearity and non-normality). The scale has demonstrated adequate validity and reliability [22-24].

To identify clusters of individuals (trajectory groups) who have followed a similar developmental trajectory for social support over the 32-year follow-up period, we also used group-based trajectory modelling (GBTM) [25]. GBTM is increasingly being applied in clinical research to map the developmental course of health-related factors and to identify the number, shape and size of different (latent) trajectory groups in the data [26]. 


\section{Covariates and Potential Mediators}

\section{Body Mass Index}

BMI has been measured from baseline (1980). In the current study, we also included measurements of BMI from 2001 as a covariate (2001 was when the last social support measure was assessed) and BMI from 2008. BMI in 2008 was included as a potential mediator as it preceded the 2012 outcome measures. BMI in 1980 and in 2012 were used in the descriptive statistics only. BMI was calculated with the formula BMI = weight $(\mathrm{kg}) /[\text { height }(\mathrm{m})]^{2}$.

\section{Inflammation}

C-reactive protein (CRP) was measured in 2012 by analysing serum high sensitive (hsCRP) by an automated analyser (Olympus AU400, Olympus, USA) and a highly sensitive turbidimetric immunoassay kit ('CRP-UL' assay, Wako Chemicals, Neuss, Germany). Detection limit of the assay was $0.06 \mathrm{mg} / \mathrm{l}$. Due to a negative skew, CRP was $\log$ transformed.

\section{Youth Depression}

Depression was measured by using a modified version of the Beck Depression Inventory (BDI) in 1992 [27]. The BDI was modified to more effectively capture instances of mild depression, by only using the items in the original scale which represented the second mildest levels of depression. The modified version has been shown to better capture depressive symptoms in the general population compared to clinically oriented scales such as BDI-II [28]. This modified version consists of 21 items (e.g. 'I often feel sad') rated on a five-point scale ( $1=$ totally disagree, $5=$ totally agree). The items are summed to give a range of 21-105, with a higher score indicating higher levels of depression. The modified BDI has demonstrated adequate validity and reliability [29].

\section{Childhood Risk Factors}

Childhood cardiovascular risk factors were measured by standard clinical methods and included BMI (1980), serum insulin (1980) and glucose levels measured in 1986 (glucose not included as a covariate in the current study). Serum insulin concentrations were measured in one laboratory by using a modification of the immunoassay method of Herbert et al. [30]. Due to a negative skew, baseline insulin levels were $\log$ transformed.

\section{Socioeconomic Status in Childhood}

Information on parental socioeconomic status (SES) were obtained with questionnaires in 1980. Parental occupation and family annual income were considered indicators of SES in childhood. Parental occupation was coded from 1 to 3 ( 1 = manual, 2 = lower non-manual, 3 = upper non-manual). Family income was measured as gross annual income and grouped into eight categories ranging from 1 (corresponding to $<$ USD 3000) to 8 (corresponding to $>$ USD 22,000). These criteria correspond to the median income per household in Finland which was 12,920 USD in 1983 [31].

\section{Statistical Analyses}

We used a series of hierarchical linear regression analyses to examine the association between social support in young adulthood and fasting glucose in mid-adulthood. Model 1 adjusted for age, BMI in young adulthood and childhood SES. Model 2 adjusted for the previous covariates + youth depression. Model 3 adjusted for the previous covariates + BMI in mid-adulthood.

We also tested the association between social support in young adulthood and prediabetes (present vs. not present) in mid-adulthood by using logistic regression analyses, sequentially adjusting for the same covariates as above (models 1-3). Odds ratios (ORs) and $95 \%$ confidence intervals (CIs) were calculated to examine how the odds for prediabetes being present were associated with the social support scores. The social support and depression measures were standardized (mean $=0, \mathrm{SD}=1)$ to simplify the interpretation of the ORs. We also repeated the linear and logistic regression analyses and adjusted for baseline insulin in all the models (as a surrogate for baseline glucose).

To test for possible mediators, we employed a bootstrapping technique [32] which allowed us to test the indirect effect of social support on prediabetes and the extent to which this association was mediated by other variables. Unstandardized indirect effects were computed for each of 5000 bootstrapped samples, and bias corrected $95 \%$ CIs were computed.

Lastly, we used group-based trajectory modelling (GBTM) [25] in order to identify clusters of individuals who have followed a similar developmental trajectory across the 32year follow-up. We used the Bayesian information criteria (BIC) to evaluate model fit. In the GBTM, the BIC is always negative and the maximum (the least negative value) indicates the best model. We then repeated the linear regression analysis by using the social support trajectory groups (as a categorical predictor) to predict fasting glucose in mid-adulthood, by using the same three models as described above.

Analyses were initially conducted including a sex interaction term (sex $\times$ social support) and as there were significant 
sex differences (e.g. $p=0.011$ for fasting glucose); the results are stratified by sex. All analyses were conducted on SPSS [22] except for the GBTM that was performed by using STATA [13].

\section{Results}

\section{Descriptive Statistics}

The descriptive statistics are portrayed in Table 1. The mean age of females at baseline (1980) was 10.9 years, and the mean age of males was 10.3 years, with an age range between 3 and 18 years. When outcome measures were assessed in 2012 (32 years after baseline and
11 years after the last social support assessment), $2.5 \%$ of women and $1.5 \%$ of men had type 2 diabetes, and $15.8 \%$ of women and $32.4 \%$ of men had type 2 diabetes or prediabetes. Mean BMI for women in 2012 was 26.2 (SD 5.5) and 26.7 (SD 4.3) for men (BMI >25 is classified as overweight).

When examining correlations between key variables, we found that for women, social support in young adulthood was correlated with BMI in 2008 but not BMI in 2001 ( $r=-0.121$ vs. $r=-0.074)$. Social support was also correlated with fasting glucose in $2012(r=-0.166)$ and youth depression $(r=-0.455)$ in women. Social support in men was only correlated with youth depression $(r=-0.373)$. Social support was not associated with future CRP in either sex $(r=-0.047$ for women and $r=0.018$ for men).

Table 1 Descriptive statistics of study sample stratified by sex $(n=1250)$

\begin{tabular}{|c|c|c|c|c|c|c|c|c|}
\hline & \multicolumn{4}{|c|}{ Females } & \multicolumn{4}{|c|}{ Males } \\
\hline & $n$ & $n(\%)$ & Mean (SD) & Range & $n$ & $n(\%)$ & Mean (SD) & Range \\
\hline \multicolumn{9}{|l|}{ Baseline variables (1980) } \\
\hline Age (years) & 722 & & $10.9(5.0)$ & $3-18$ & 528 & & $10.3(5.1)$ & $3-18$ \\
\hline Parental income (gross annual income) & 722 & & & & 528 & & & \\
\hline Level 1 (lowest income bracket) & & $38(5.3 \%)$ & & & & $18(3.4 \%)$ & & \\
\hline Level 2 & & $59(8.2 \%)$ & & & & $35(6.6 \%)$ & & \\
\hline Level 3 & & $85(11.8 \%)$ & & & & $65(12.3 \%)$ & & \\
\hline Level 4 & & $105(14.5 \%)$ & & & & $79(15.0 \%)$ & & \\
\hline Level 5 & & $113(15.7 \%)$ & & & & $84(15.9 \%)$ & & \\
\hline Level 6 & & $154(21.3 \%)$ & & & & $125(23.7 \%)$ & & \\
\hline Level 7 & & $108(15.0 \%)$ & & & & $75(14.2 \%)$ & & \\
\hline Level 8 (highest income bracket) & & $60(8.3 \%)$ & & & & $47(8.9 \%)$ & & \\
\hline Parental occupational status & 722 & & & & 528 & & & \\
\hline Manual & & $170(23.5 \%)$ & & & & $149(28.2 \%)$ & & \\
\hline Lower non-manual & & $445(61.6 \%)$ & & & & $309(58.5 \%)$ & & \\
\hline Upper non-manual & & $107(14.8 \%)$ & & & & $70(13.3 \%)$ & & \\
\hline BMI $\left(\mathrm{kg} / \mathrm{m}^{2}\right)$ & 720 & & $17.9(3.0)$ & & 523 & & $17.8(2.9)$ & \\
\hline Insulin (transformed) ${ }^{\mathrm{a}}$ & 722 & & $0.99(0.3)$ & & 528 & & $0.90(0.3)$ & \\
\hline \multicolumn{9}{|l|}{ Youth/young adulthood variables } \\
\hline Depression (1992) & 722 & & $46.3(12.6)$ & $21-105$ & 528 & & $41.9(11.2)$ & $21-105$ \\
\hline $\begin{array}{l}\text { Social support (transformed })^{\mathrm{a}} \\
\text { BMI }\left(\mathrm{kg} / \mathrm{m}^{2}\right)(2001) \\
\text { Mid-adulthood variables }\end{array}$ & $\begin{array}{l}722 \\
722\end{array}$ & & $\begin{array}{r}5.0(1.0) \\
24.4(4.6)\end{array}$ & & $\begin{array}{l}528 \\
528\end{array}$ & & $\begin{array}{r}4.2(1.0) \\
25.6(3.8)\end{array}$ & \\
\hline $\begin{array}{l}\text { BMI }\left(\mathrm{kg} / \mathrm{m}^{2}\right)(2008) \\
\text { BMI }\left(\mathrm{kg} / \mathrm{m}^{2}\right)(2012) \\
\text { CRP (transformed) }(2012)^{\mathrm{a}}\end{array}$ & $\begin{array}{l}722 \\
722 \\
722\end{array}$ & & $\begin{array}{r}25.4(5.0) \\
26.2(5.5) \\
-0.2(1.3)\end{array}$ & & $\begin{array}{l}528 \\
528 \\
528\end{array}$ & & $\begin{array}{r}26.4(4.0) \\
26.7(4.3) \\
-0.3(1.1)\end{array}$ & \\
\hline Fasting glucose (mmol/l) (2012) & 722 & & $5.2(0.8)$ & & 528 & & $5.5(0.7)$ & \\
\hline Type 2 diabetes (2012) & 722 & $18(2.5 \%)$ & & & 528 & $8(1.5 \%)$ & & \\
\hline Prediabetes or type 2 diabetes (2012) & 722 & $114(15.8 \%)$ & & & 528 & $171(32.4 \%)$ & & \\
\hline
\end{tabular}

Social support was the average of four measurements taken in youth and young adulthood (1989, 1992, 1997 and 2001)

$B M I$ body mass index, $C R P C$-reactive protein

${ }^{a}$ Insulin and C-reactive protein were transformed by using $\log$ transformations, and social support was transformed by using a square root transformation 


\section{Social Support, Fasting Glucose and Prediabetes}

Social support was a statistically significant predictor of fasting glucose for women in the first two models which adjusted for SES and youth depression $(\beta=-0.144, p<0.001$; $\beta=-0.136, p=0.001$ ) but not in the fully adjusted model which also adjusted for BMI in 2008 ( $p=0.400$; Table 2). In contrast, social support was not a significant predictor of fasting glucose for men in any of the models (see Table 2).

Logistic regression results are presented in Table 3. Lower social support in young adulthood was associated with higher odds of prediabetes in mid-adulthood in women, but not men. This association in women remained robust after adjusting for SES in model 1 ( $\beta=1.31,95 \%$ CI 1.02 to $1.69, p=0.032)$, but not after additional adjustment for depression $(p=0.234)$ or in the fully adjusted model which also adjusted for BMI in $2008(p=0.560)$. For men, the only significant predictor in the fully adjusted model was parental income $(p=0.035)$.

We repeated the regression analyses and adjusted for baseline insulin in all the models as a surrogate for baseline glucose. The findings did not change (results not tabulated). We also tested the collinearity diagnostics of the models which included BMI in 2001 and 2008, due to the high correlation between these two variables $(r=0.88)$. All the variance inflation factors (VIFs) were well below 10, and the tolerance statistics were all above 0.2 , indicating that there was no collinearity within the data (VIF $<2$; tolerance $>0.60$ ).

\section{Mediation Analyses}

In Table 4, we present data from the multiple mediation model, where we examined whether BMI in 2008 or youth depression were mediating the relationship between social support and prediabetes. We tested the significance of the indirect effect by using bootstrapping procedures.

We found evidence that as a set, BMI and youth depression were mediating the relationship between social support and prediabetes ( $\beta$ for total indirect effect $\beta=0.20, \mathrm{SE}=0.07$ ). Examining the specific indirect effects showed that only BMI was mediating the relationship, as the $95 \% \mathrm{CI}$ did not contain zero ( $\beta$ for specific indirect effect $\beta=0.09, \mathrm{SE}=0.03,95 \%$ $\mathrm{CI}=0.03$ to 0.16 ). In contrast, there was little evidence that youth depression was a mediator $(\beta$ for specific indirect effect $\beta=0.11, \mathrm{SE}=0.06,95 \% \mathrm{CI}=-0.01$ to 0.23 ).

\section{Social Support Trajectory Groups}

Lastly, we repeated the linear regression analysis to examine the association between the social support trajectory groups and fasting glucose in 2012 by using the same three models as previously (see Table 5). As shown in Fig. 2, the social support trajectories from baseline to 2012 based on GBTM reflected patterns of low, moderate and high levels of social support over time. Based on their individual patterns, they were defined under the following four categories: low and decreasing social support (group 1), low but increasing social support (group 2), moderate to high social support (group 3) and consistently high social support (group 4).

As expected, we found no significant association for men (results not tabulated). For women, we found that compared with the group with low and decreasing social support over time (group 1, reference group), all the other groups were negatively associated with fasting glucose in mid-adulthood, even after adjusting for age, SES, BMI in 2001 and depressive

Table 2 Age-adjusted beta coefficients of social support in young adulthood as a predictor of glucose levels in mid-adulthood for women and men, sequentially adjusting for SES, depression and BMI (2008)

\begin{tabular}{|c|c|c|c|c|c|c|}
\hline & \multicolumn{3}{|l|}{ Women } & \multicolumn{3}{|l|}{ Men } \\
\hline & Model 1 & Model 2 & Model 3 & Model 1 & Model 2 & Model 3 \\
\hline Predictors & $\beta^{\mathrm{a}}(p$ value $)$ & $\beta$ ( $p$ value $)$ & $\beta$ ( $p$ value $)$ & $\beta^{\mathrm{a}}$ ( $p$ value $)$ & $\beta$ ( $p$ value $)$ & $\beta$ ( $p$ value $)$ \\
\hline Perceived social support & $-0.144(p<0.001)$ & $-0.136(p=0.001)$ & $-0.036(p=0.400)$ & $-0.021(p=0.642)$ & $-0.050(p=0.301)$ & $-0.059(p=0.241)$ \\
\hline Parental income & $-0.048(p=0.253)$ & $-0.049(p=0.244)$ & $-0.047(p=0.294)$ & $-0.012(p=0.817)$ & $-0.013(p=0.801)$ & $-0.003(p=0.958)$ \\
\hline \multicolumn{7}{|l|}{ Parental occupation } \\
\hline Manual & $0.008(p=0.897)$ & $0.009(p=0.874)$ & $-0.005(p=0.930)$ & $-0.076(p=0.296)$ & $-0.076(p=0.295)$ & $-0.083(p=0.266)$ \\
\hline Lower non-manual & $0.053(p=0.317)$ & $0.054(p=0.309)$ & $0.016(p=0.773)$ & $0.036(p=0.585)$ & $0.035(p=0.601)$ & $0.029(p=0.667)$ \\
\hline Upper non-manual & Reference & Reference & Reference & Reference & Reference & Reference \\
\hline BMI in 2001 & $0.290(p<0.001)$ & $0.288(p<0.001)$ & $-0.057(p=0.480)$ & $0.183(p<0.001)$ & $0.195(p<0.001)$ & $-0.040(p=0.696)$ \\
\hline Youth depression & & $0.018(p=0.649)$ & $0.061(p=0.153)$ & & $-0.079(p=0.107)$ & $-0.094(p=0.067)$ \\
\hline BMI in 2008 & & & $0.357(p<0.001)$ & & & $0.273(p=0.008)$ \\
\hline
\end{tabular}

The bold values are significant at $p<0.05$. All variables in each model are entered simultaneously

${ }^{a}$ Standardized beta coefficients 
Table 3 Age-adjusted odds ratios and $95 \%$ CIs for low social support in young adulthood predicting prediabetes in mid-adulthood in women and men, sequentially adjusting for SES, depression and BMI (2008)

\begin{tabular}{|c|c|c|c|c|c|c|c|c|c|c|c|c|}
\hline \multirow[b]{3}{*}{ Predictors } & & \multicolumn{5}{|l|}{ Women } & \multicolumn{5}{|c|}{ Men } & \\
\hline & \multicolumn{2}{|c|}{ Model 1} & \multicolumn{2}{|c|}{ Model 2} & \multicolumn{2}{|c|}{ Model 3} & \multicolumn{2}{|c|}{ Model 1} & \multicolumn{2}{|c|}{ Model 2} & \multicolumn{2}{|c|}{ Model 3} \\
\hline & OR & $95 \% \mathrm{CI}$ & OR & $95 \% \mathrm{CI}$ & OR & $95 \% \mathrm{CI}$ & OR & $95 \% \mathrm{CI}$ & OR & $95 \% \mathrm{CI}$ & OR & $95 \% \mathrm{CI}$ \\
\hline Low social support & 1.31 & 1.02 to 1.69 & 1.18 & 0.90 to 1.56 & 1.10 & 0.81 to 1.49 & 0.97 & 0.77 to 1.21 & 1.03 & 0.80 to 1.31 & 1.09 & 0.84 to 1.41 \\
\hline Parental income & 1.08 & 0.94 to 1.22 & 1.07 & 0.94 to 1.22 & 1.05 & 0.91 to 1.22 & 0.90 & 0.80 to 1.02 & 0.90 & 0.80 to 1.02 & 0.87 & 0.76 to 0.99 \\
\hline \multicolumn{13}{|l|}{ Parental occupation } \\
\hline Manual & 0.77 & 0.32 to 1.83 & 0.81 & 0.34 to 1.94 & 1.08 & 0.42 to 2.80 & 0.76 & 0.37 to 1.56 & 0.75 & 0.37 to 1.56 & 0.70 & 0.32 to 1.50 \\
\hline Lower non-manual & 1.03 & 0.54 to 1.95 & 1.06 & 0.55 to 2.02 & 0.98 & 0.49 to 1.99 & 0.87 & 0.49 to 1.55 & 0.87 & 0.49 to 1.54 & 0.94 & 0.52 to 1.71 \\
\hline Upper non-manual & & Reference & & Reference & & Reference & & Reference & & Reference & & Reference \\
\hline BMI in 2001 & 1.15 & 1.10 to 1.20 & 1.15 & 1.10 to 1.20 & 0.89 & 0.80 to 0.99 & 1.08 & 1.02 to 1.13 & 1.08 & 1.03 to 1.14 & 0.96 & 0.85 to 1.09 \\
\hline Youth depression & & & 1.24 & 0.98 to 1.58 & 1.32 & 1.01 to 1.72 & & & 0.86 & 0.67 to 1.10 & 0.84 & 0.65 to 1.09 \\
\hline BMI in 2008 & & & & & 1.31 & 1.18 to 1.45 & & & & & 1.12 & 0.99 to 1.26 \\
\hline
\end{tabular}

The bold values are significant at $p<0.05$. All variables in each model are entered simultaneously. ORs associated with a one standard deviation increase in social support. Prediabetes and type 2 diabetes cases were combined

symptoms (models 1 and $2, p<0.01$ ). After adjusting for BMI in 2008 (model 3), this association was no longer significant.

\section{Discussion}

Recently, evidence has started to accrue that many psychosocial factors which are associated with cardiovascular disease, such as social support, are also implicated in type 2 diabetes. Our results support these findings and suggest that low social support is associated with higher fasting glucose in both sexes (although less pronounced in men) and increases the risk of prediabetes (and possibly type 2 diabetes) in women. Furthermore, our findings indicate that for women, this association is mediated through BMI.

Table 4 Mediation of the effect of social support on prediabetes through BMI and depression, adjusting for age

\begin{tabular}{lcrrr}
\hline Possible mediators & \multicolumn{4}{c}{ Bootstrap results for indirect effects $(95 \%$ CI) } \\
\cline { 2 - 5 } & Coefficient $^{\mathrm{a}}$ & SE & Lower CI & Upper CI \\
\hline BMI (2008) & 0.0892 & 0.0346 & $\mathbf{0 . 0 2 8 1}$ & $\mathbf{0 . 1 6 4 8}$ \\
Youth depression (1992) & 0.1145 & 0.0595 & -0.0051 & 0.2258 \\
Total & 0.2037 & 0.0671 & $\mathbf{0 . 0 6 9 3}$ & $\mathbf{0 . 3 3 2 6}$ \\
Contrast & -0.0252 & 0.0705 & -0.1627 & 0.1163 \\
\hline
\end{tabular}

Sample includes females only $(n=643)$. The number of bootstrap resamples is 5000. The bold values indicate that the CI does not include zero, thus meeting criteria for mediation [32]

${ }^{\text {a }}$ Mean of the indirect effect estimates calculated across bootstrap samples
Our results did not find any support for a pathway involving stress-related inflammation, as CRP (an inflammatory marker associated with chronic stress and diabetes) was not associated with social support for either sex. Furthermore, we did not find any evidence that depression, which is closely associated with stress-related inflammation, was mediating the relationship between social support and prediabetes in women.

The mediating role of BMI was as anticipated, given that BMI is considered the most important risk factor for type 2 diabetes in women [33]. Previous studies have found an association between social support and certain health behaviours (in particular diet and exercise), factors which protect against obesity and prediabetes. For example, high levels of social support have been associated with health-promoting behaviours like eating a healthy diet [19], as well as maintaining physical activity [34]. In future research, we hope to examine the role of health behaviours, in order to further tease apart the relationship between social support, health behaviours/BMI and the impact on prediabetes.

There are several possible explanations for why perceived social support predicted prediabetes in women but not in men. It is possible that the sex differences were due to the aspects of social relations we measured (functional rather than structural). Research has generally found that men and women not only have different types of social networks (e.g. women tend to have more confidants, while men have wider, less closeknit networks) but they also value different types of social support [35]. As well as valuing different sources of social support, the quality of the relationship and marital functioning seems to play a stronger role for women's health outcomes in comparison to men [36]. Due to these sex differences, and 
because we measured functional social support rather than structural, we perhaps only tapped into the aspects of social relations that are beneficial for women.

Previous evidence has also suggested that the social determinants of cardiometabolic disorders may differ by sex and that the effect of psychosocial factors on type 2 diabetes might be stronger in women [37]. It is thus possible that social support has a different health impact on women compared to men and that low social support may be more detrimental to women's health. Studies have found that women may be more vulnerable to the effects of psychosocial stress than men, with more pronounced neuroendocrine responses to chronic stress [38], which may be true for low social support as well. For example, low social support may lead to a stronger stress response or to a more maladaptive coping strategy (e.g. eating unhealthily), thereby leading to stress-related inflammation or weight gain. There is also the possible interaction between age of menarche/menopause, hormones (including cortisol, oxytocin as well as sex-specific hormones) and social support which we were not able to take fully into account. For example, biobehavioural differences in stress responses in men and women may also be implicated in the association between low social support and adverse health outcomes [39]. These are all avenues which warrant further study, to clarify sex-specific effects of social support and its impact on health outcomes.

Previous studies have found that childhood SES is associated with cardiometabolic conditions including type 2 diabetes [40]. Furthermore, children from lower SES backgrounds have lower levels of social support [41]. In our study, the association between social support and both fasting glucose and prediabetes in women remained after adjusting for childhood SES, suggesting that the findings are independent of SES. In contrast, in the fully adjusted model for men, the only significant predictor of prediabetes was parental income. We intend to verify these findings by adding cumulative measures of SES across the life span, to determine whether the association between social

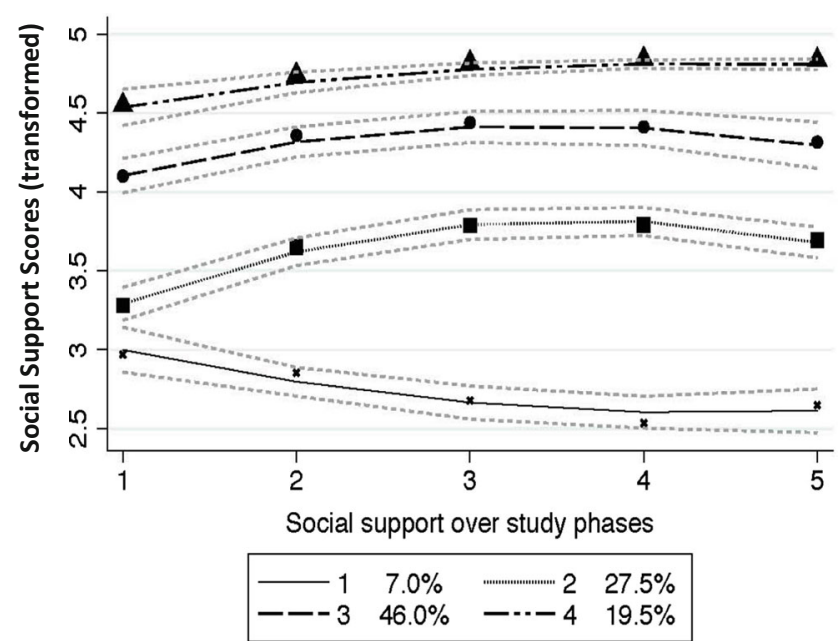

Fig. 2 Social support trajectories across the study phases. Shown are the results from the group-based trajectory modelling which found a fourtrajectory solution, reflecting patterns of low, moderate and high levels of social support over time. The four social support trajectories are depicted with $95 \%$ CIs (grey dotted lines)

support and prediabetes remains after adjusting for SES in adulthood and whether this differs by sex.

When we examined the association between the social support trajectory groups and fasting glucose in 2012, we found that compared to group 1 (low and decreasing social support over time), the other groups were negatively associated with fasting glucose in mid-adulthood. Once BMI (in 2008) was adjusted for, the social support trajectories were no longer associated with fasting glucose, once again supporting the prominent role that BMI seems to play in this relationship. In future research, we intend to further explore the temporal relationship between the social support trajectories and fasting glucose by using growth curve models, in order to further examine how social support impacts on glucose levels over time.

Future research should also examine the contribution of both structural and function social support and its association with future risk of type 2 diabetes. Importantly, although both structural and functional social support has been associated

Table 5 Age-adjusted beta coefficients of the four social support trajectory groups and glucose levels in mid-adulthood in women $(n=677)$, sequentially adjusting for SES, depression and BMI (2008)

\begin{tabular}{llll}
\hline & Model 1 & Model 2 & Model 3 \\
\hline Predictors & $\beta^{\text {a }}(p$ value $)$ & $\beta(p$ value $)$ & $\beta(p$ value $)$ \\
Low and decreasing social support (group 1) & Reference & Reference & Reference \\
Low but increasing social support (group 2) & $-\mathbf{0 . 3 2 6}(\boldsymbol{p}<\mathbf{0 . 0 0 1 )}$ & $\mathbf{- 0 . 3 2 0}(\boldsymbol{p}=\mathbf{0 . 0 0 1})$ & $-0.011(p=0.912)$ \\
Moderate to high social support (group 3) & $\mathbf{- 0 . 4 5 6}(\boldsymbol{p}<\mathbf{0 . 0 0 1 )}$ & $\mathbf{- 0 . 4 3 2}(\boldsymbol{p}<\mathbf{0 . 0 0 1 )}$ & $0.012(p=0.927)$ \\
Consistently high social support (group 4) & $\mathbf{- 0 . 4 2 5}(\boldsymbol{p}<\mathbf{0 . 0 0 1 )}$ & $\mathbf{- 0 . 3 9 2}(\boldsymbol{p}<\mathbf{0 . 0 0 1 )}$ & $0.001(p=0.995)$ \\
\hline
\end{tabular}

The bold values are significant at $p<0.05$. All variables in each model are entered simultaneously. Model 1 adjusted for age, SES and BMI in 2001; model 2 adjusted for the previous variables and depressive symptoms; model 3 adjusted for the previous variables and BMI in 2008

${ }^{\mathrm{a}}$ Standardized beta coefficients 
with improved health outcomes, the underlying pathways through which they influence health outcomes may not necessarily represent independent pathways [42, 43]. This remains a question for future research, including exploring the possible differences between functional and structural social support and their influence on health outcomes.

Strengths of our study include a prospective, populationbased design which attempted to not only clarify the link between social support and prediabetes but also to explore the temporal relationships underlying this relationship by using a prospective study design and taking into account a large set of potential confounders, starting from childhood. The mechanisms between social factors and disease outcomes such as type 2 diabetes are still unclear [44], and the current study is an attempt to establish the mechanisms leading from low social support to prediabetes. To our knowledge, this is the first study to use mediation analysis to examine the pathways between social support and prediabetes.

The following limitations should be noted. Similar to most longitudinal studies, there has been loss of participants across the follow-ups. However, we have previously demonstrated that the current cohort is representative of the original sample [45]. Another limitation was that our sample was drawn from a homogeneous population with respect to ethnicity and SES; therefore, the results may not generalize cross-culturally. Another limitation was that we did not assess health behaviours and their role in the relationship between social support, BMI and prediabetes. Also, rather than assessing social support over four-time points from youth to young adulthood, we averaged the data to form a single measure of social support in young adulthood. However, evidence suggests that perceived social support tends to remain relatively stable over time [46]. Moreover, when we repeated the analyses by using the social support trajectories as a predictor of fasting glucose, we found similar results. We also did not examine whether depression and social support have a bidirectional relationship, whereby depression is driving low social support and not vice versa. Lastly, in our mediation models, we only controlled for age, so it is possible there were other confounders that should have been included in the models.

In summary, our results suggest that low perceived social support in young adulthood increases the risk for high fasting glucose and prediabetes in mid-adulthood in women. The association between social support and prediabetes in women was partially mediated by BMI, supporting the prominent role that BMI plays in the development of prediabetes. Recently, there have been calls to examine the mechanisms between social factors and health outcomes [44]. Our findings contribute to this literature by highlighting the importance of BMI as a mediational pathway between social support and prediabetes.
Acknowledgments We greatly acknowledge Irina Lisinen, Johanna Ikonen and Ville Aalto for assistance in managing the Cardiovascular Risk in Young Finns dataset.

\section{Compliance with Ethical Standards}

Funding The Young Finns Study has been financially supported by the Academy of Finland: grants 126925, 121584, 124282, 129378 (Salve), 117787 (Gendi), 41071 (Skidi), 265869, 258711, 258578, 265977 and 286284; the Social Insurance Institution of Finland, Kuopio, Tampere and Turku University Hospital Medical Funds (grants 9N035 and X51001), the Juho Vainio Foundation, the Signe and Ane Gyllenberg Foundation, the Emil Aaltonen Foundation, the Paavo Nurmi Foundation, the Finnish Foundation of Cardiovascular Research, the Finnish Cultural Foundation, the Osk. Huttunen Foundation and the Tampere Tuberculosis Foundation.

Conflict of Interest No conflict of interest declared.

Ethical Approval All procedures performed in studies involving human participants were in accordance with the ethical standards of the institutional and/or national research committee and with the 1964 Helsinki Declaration and its later amendments or comparable ethical standards.

Informed Consent Informed consent was obtained from all individual participants included in the study.

\section{References}

1. Wild S, Roglic G, Green A, Sicree R, King H. Global prevalence of diabetes: estimates for the year 2000 and projections for 2030 . Diabetes Care. 2004;27(5):1047-53.

2. Bullard KM, Saydah SH, Imperatore G, Cowie CC, Gregg EW, Geiss LS, et al. Secular changes in U.S. prediabetes prevalence defined by hemoglobin A1c and fasting plasma glucose: National Health and nutrition examination surveys, 1999-2010. Diabetes Care. 2013;36(8):2286-93.

3. Nathan DM, Davidson MB, DeFronzo RA, Heine RJ, Henry RR, Pratley R, et al. Impaired fasting glucose and impaired glucose tolerance: implications for care. Diabetes Care. 2007;30(3):753-9.

4. Evans JM, Newton RW, Ruta DA, MacDonald TM, Morris AD. Socio-economic status, obesity and prevalence of type 1 and type 2 diabetes mellitus. Diabet Med. 2000;17(6):478-80.

5. Knol MJ, Twisk JWR, Beekman ATF, Heine RJ, Snoek FJ, Pouwer F. Depression as a risk factor for the onset of type 2 diabetes mellitus. A meta-analysis. Diabetologia. 2006;49(5):837-45.

6. Heraclides A, Chandola T, Witte DR, Brunner EJ. Psychosocial stress at work doubles the risk of type 2 diabetes in middle-aged women: evidence from the Whitehall II study. Diabetes Care. 2009;32(12):2230-5.

7. Lett HS, Blumenthal JA, Babyak MA, Strauman TJ, Robins C, Sherwood A. Social support and coronary heart disease: epidemiologic evidence and implications for treatment. Psychosom Med. 2005;67(6):869-78.

8. Hilding A, Shen C, Östenson C-G. Social network and development of prediabetes and type 2 diabetes in middle-aged Swedish women and men. Diabetes Res Clin Pract. 2015;107(1):166-77.

9. Meisinger C, Kandler U, Ladwig K-H. Living alone is associated with an increased risk of type 2 diabetes mellitus in men but not 
women from the general population: the MONICA/KORA Augsburg cohort study. Psychosom Med. 2009;71(7):784-8.

10. Thoits PA. Mechanisms linking social ties and support to physical and mental health. J Health Soc Behav. 2011;52(2):145-61.

11. Rosmond R. Stress induced disturbances of the HPA axis: a pathway to type 2 diabetes? Med Sci Monit. 2003;9(2):RA35-9.

12. Grant N, Hamer M, Steptoe A. Social isolation and stress-related cardiovascular, lipid, and cortisol responses. Ann Behav Med. 2009;37(1):29-37.

13. Wang ML, Pbert L, Lemon SC. Influence of family, friend and coworker social support and social undermining on weight gain prevention among adults. Obesity (Silver Spring). 2014;22(9): 1973-80.

14. Wing RR, Matthews KA, Kuller LH, Meilahn EN, Plantinga P. Waist to hip ratio in middle-aged women. Associations with behavioral and psychosocial factors and with changes in cardiovascular risk factors. Arterioscler Thromb Vasc Biol. 1991;11(5):1250-7.

15. Midei AJ, Matthews KA. Social relationships and negative emotional traits are associated with central adiposity and arterial stiffness in healthy adolescents. Health Psychol. 2009;28(3):347-53.

16. Aneshensel CS, Stone JD. Stress and depression: a test of the buffering model of social support. Arch Gen Psychiatry. 1982;39(12): 1392-6.

17. Thoits PA. Stress, coping, and social support processes: where are we? What next? J Health Soc Behav. 1995:53-79.

18. Aspinwall LGA. Stitch in time: self-regulation and proactive coping. Psychol Bull. 1997;121(3):417-36.

19. Emmons KM, Barbeau EM, Gutheil C, Stryker JE, Stoddard AM. Social influences, social context, and health behaviors among working-class, multi-ethnic adults. Health Educ Behav. 2007;34(2):315-34.

20. Raitakari OT, Juonala M, Rönnemaa T, Keltikangas-Järvinen L, Räsänen L, Pietikäinen M, et al. Cohort profile: the cardiovascular risk in young Finns study. Int J Epidemiol. 2008;37(6):1220-6.

21. Alberti KG, Zimmet P, Shaw J. The metabolic syndrome: a new world-wide definition. Lancet. 2005;366:1059-62.

22. Blumenthal JA, Burg MM, Barefoot J, Williams RB, Haney T, Zimet G. Social support, type a behavior, and coronary artery disease. Psychosom Med. 1987;49(4):331-40.

23. Ravaja N, Keltikangas-Järvinen L, Viikari J. Perceived social support and abdominal fat distribution in adolescents and young adults: a structural equation analysis of prospective data. Appetite. 1998;31(1):21-35.

24. Keltikangas-Järvinen L, Ravaja N. Relationships between hostility and physiological coronary heart disease risk factors in young adults: moderating influence of perceived social support and sociability. Psychol Health. 2002;17(2):173-90.

25. Nagin DS, Tremblay RE. Analyzing developmental trajectories of distinct but related behaviors: a group-based method. Psychol Methods. 2001;6(1):18-34.

26. Kaup AR, Byers AL, Falvey C, Simonsick EM, Satterfield S, Ayonayon HN, et al. Trajectories of depressive symptoms in older adults and risk of dementia. JAMA psychiatry. 2016.

27. Katainen S, Räikkönen K, Keltikangas-Järvinen L. Adolescent temperament, perceived social support, and depressive tendencies as predictors of depressive tendencies in young adulthod. Eur J Pers. 1999;13:183-207.

28. Rosenström T, Jokela M, Puttonen S, Hintsanen M, Pulkki-Råback L, Viikari JS, et al. Pairwise measures of causal direction in the epidemiology of sleep problems and depression. PLoS One. 2012;7(11) e50841.
29. Elovainio M, Keltikangas-Järvinen L, Pulkki-Råback L, Kivimäki M, Puttonen S, Viikari L, et al. Depressive symptoms and c-reactive protein: the cardiovascular risk in Young Finns Study. Psychol Med. 2006;36(6):797-805.

30. Herbert V, Lau KS, Gottlieb CW, Bleicher SJ. Coated charcoal immunoassay of insulin. J Clin Endocrinol Metab. 1965;25(10):1375-84.

31. Statistics Finland. Distribution of household income in Finland. Helsinki: Statistics Finland, 1983.

32. Preacher KJ, Hayes AFSPSS. SAS procedures for estimating indirect effects in simple mediation models. Behavior Research Methods, Instruments, and Computers. 2004;36:717-31.

33. Hu FB, Manson JE, Stampfer MJ, Colditz G, Liu S, Solomon CG, et al. Diet, lifestyle, and the risk of type 2 diabetes mellitus in women. N Engl J Med. 2001;345(11):790-7.

34. Kouvonen A, De Vogli R, Stafford M, Shipley MJ, Marmot MG, Cox T, et al. Social support and the likelihood of maintaining and improving levels of physical activity: the Whitehall II Study. Eur J Public Health. 2011:ckr091.

35. Flaherty J, Richman J. Gender differences in the perception and utilization of social support: theoretical perspectives and an empirical test. Social Sciences and Medicine. 1989;28:1221-122.

36. Robles TF, Slatcher RB, Trombello JM, McGinn MM. Marital quality and health: a meta-analytic review. Psychol Bull. 2014;140(1):140-87.

37. Heraclides AM, Chandola T, Witte DR, Brunner EJ. Work stress, obesity and the risk of type 2 diabetes: gender-specific bidirectional effect in the Whitehall II study. Obesity. 2012;20(2):428-33.

38. Rohleder N, Schommer NC, Hellhammer DH, Engel R, Kirschbaum C. Sex differences in glucocorticoid sensitivity of Proinflammatory cytokine production after psychosocial stress. Psychosom Med. 2001;63(6):966-72.

39. Taylor SE, Klein LC, Lewis BP, Gruenewald TL, Gurung RAR, Updegraff JA. Biobehavioral responses to stress in females: tendand-befriend, not fight-or-flight. Psychol Rev. 2000;107(3):411.

40. Slopen N, Goodman E, Koenen KC, Kubzansky LD. Socioeconomic and other social stressors and biomarkers of cardiometabolic risk in youth: a systematic review of less studied risk factors. PLoS One. 2013;8(5):e64418.

41. Evans GW. The environment of childhood poverty. Am Psychol. 2004;59(2):77.

42. Uchino BN. Social support and health: a review of physiological processes potentially underlying links to disease outcomes. J Behav Med. 2006;29(4):377-87.

43. Hakulinen C, Pulkki-Råback L, Jokela M, E Ferrie J, Aalto A-M, Virtanen M, et al. Structural and functional aspects of social support as predictors of mental and physical health trajectories: Whitehall II cohort study. J Epidemiol Community Health. 2016;2016.

44. Havranek EP, Mujahid MS, Barr DA, Blair IV, Cohen MS, CruzFlores S, et al. Social determinants of risk and outcomes for cardiovascular disease: a scientific statement from the American Heart Association. Circulation. 2015;2015.

45. Juonala M, Juhola J, Magnussen CG, Wurtz P, Viikari JS, Thomson $\mathrm{R}$, et al. Childhood environmental and genetic predictors of adulthood obesity: the cardiovascular risk in young Finns study. J Clin Endocrinol Metab. 2011;96(9):E1542-E9.

46. Shaw BA, Krause N, Chatters LM, Connell CM, Ingersoll-Dayton B. Emotional support from parents early in life, aging, and health. Psychol Aging. 2004;19(1):4-12. 